\title{
Aplicação de Algoritmos Combinatórios para o Problema do Caminho Tropical em Grafos
}

\author{
Igor de M. Sampaio ${ }^{1}$, Karla R. P. S. Lima ${ }^{1}$ \\ ${ }^{1}$ Escola de Artes, Ciências e Humanidades (EACH) - Universidade de São Paulo (USP) \\ São Paulo - SP - Brasil \\ \{igorms, ksampaiolima\}@usp.br
}

\begin{abstract}
The problem of the tropical path in graphs, TPP, consists of given a graph $G$ and a coloring of the vertices of $G$, finding a colored path $P$ in $G$ where each color used by $G$ appears exactly once in $P$. In this project we propose to investigate two versions of optimization of this problem: STTP, Shortest tropical path problem, that consists of finding a tropical path of minimum weight, such that the weights are assigned to the edges of $G$, and the MTPP, Maximum tropical problem path, that consists of finding the path with the largest number of colors used by G. It is known that the problem is NP-hard for graphs in general and some other variants of the problem. From the point of view of polyhedral combinatorics, there are numerous modeling techniques that allow to find optimal solutions to difficult problems in terms of computational complexity. Therefore, the objective of this project is to implement a good formulation for the problem and to associate it with high performance algorithms to obtain optimal solutions for large instances.
\end{abstract}

Resumo. O problema do caminho tropical em grafos, TPP, consiste em, dado um grafo $G$ e uma coloração dos vértices de $G$, encontrar um caminho colorido $P$ em $G$ em que cada cor usada por $G$ apareça exatamente uma vez em $P$. Neste projeto propomos investigar duas versões de otimização desse problema: STPP, o problema do caminho tropical mínimo, que consiste em encontrar um caminho tropical de peso mínimo em que os pesos são atribuídos às arestas de G, e o MTPP, o problema do caminho tropical máximo, que consiste em encontrar o caminho com o maior número de cores usadas por G. É sabido que o problema é NP-difícil para grafos em geral e algumas outras variantes do problema. Do ponto de vista da combinatória poliédrica existem inúmeras técnicas de modelagem que permitem encontrar soluções ótimas para problemas dessa natureza em termos da complexidade computacional. Diante disso, o objetivo desse projeto é implementar uma boa formulação para o problema e associar algoritmos de alto desempenho para obtenção de soluções ótimas para instâncias de grande porte.

\section{Introdução}

Qual é a rota mais rápida entre duas cidades? Em que ordem um vendedor ambulante deve visitar cidades para minimizar o tempo de viagem? De quantas camadas um chip de computador precisa para que os fios da mesma camada não se atravessem? [West et al. 2001] Esses são alguns dos inúmeros problemas do mundo real que podem ser descritos por 
meio de um diagrama que consiste em um conjunto de pontos juntamente com linhas que unem certos pares desses pontos. Por exemplo, os pontos podem representar as cidades que o vendedor ambulante quer visitar e as linhas pares de cidades vizinhas. De acordo com a teoria dos grafos, esses diagramas podem ser representados por grafos onde os pontos são chamados de vértices e as linhas de arestas. A teoria dos grafos é uma linha de pesquisa da ciência da computação teórica que possui vasta aplicação na modelagem de problemas do mundo real.

Neste trabalho estudamos mais especificamente grafos coloridos nos vértices, ou seja, são grafos em que cada vértice é atribuído uma cor. Tal atribuição não obedece nenhuma definição clássica de coloração de grafos, consiste em uma simples atribuição de cores [West et al. 2001]. Este tipo de grafo é aplicado em inúmeros problemas do mundo real, um exemplo muito conhecido é o quebra cabeça Sudoku. Outras importantes aplicações que podem ser citadas são agendamento de voo de aeronaves, tarefas entre dois processadores (presente em agendamento de transferências de arquivos entre processadores ou no caso de testes de diagnóstico mútuo de processadores) [Marx 2004], prevenção de conflitos (um exemplo seria a separação de produtos com risco de explosão), atribuição de frequências de rádio [Bondy et al. 1976], na bioinformática (pipeline de alinhamento de múltiplas sequências ou para múltiplas redes de interação proteína-proteína) [Corel et al. 2010], entre outros inúmeros problemas [Marx 2004].

O problema de caminhos tropicais consiste em uma variação do problema de ocorrência de motifs em grafos. Tal problema consiste em, dado um grafo $G=(V, A)$ e um motif $M$, onde $G$ é um grafo colorido nos vértices e $M$ é o multiconjunto de cores (uma cor pode aparecer mais de uma vez em $M$ ), encontrar um subgrafo conexo cujo conjunto de cores seja exatamente $M$. Note que para o TPP o subgrafo conexo procurado compreende um caminho e o motif é o conjunto formado por todas cores utilizadas por G.

Um caminho de um grafo colorido nos vértices é um caminho tropical sempre que conter todas as cores usadas no grafo. Para este problema existe uma versão de minimização e uma versão de maximização ambos sendo demonstrado ter classificação NP-difícil na teoria de complexidade computacional [Cohen et al. 2019]. Nosso estudo almeja a construção de um modelo robusto com o uso de técnicas da combinatória poliédrica para obter a solução ótima para instâncias de grande porte do problema. Tais técnicas envolvem programação linear inteira e a descrição de estruturas poliédricas associadas à construção de algoritmos, tais como brach-and-bound e branch-and-cut. Nossa motivação para este estudo baseia-se em todos os pontos apresentados acima, além da constatação de que não foi encontrado na literatura resultados deste problema na linha de otimização linear.

\section{Trabalhos Relacionados}

O problema de interesse originou-se inicialmente do problema de motifs em grafos, bastante investigado na atual literatura [Sikora 2012]. Relacionando ambos, a principal diferença, para uma mesma entrada do problema, consiste na solução obtida. No problema de motifs a solução é um subgrafo conexo contendo o motif enquanto que no problema do caminho tropical a solução é um caminho contendo todas as cores de grafo, duas estruturas com definições distintas. 
O principal trabalho encontrado na literatura referente a este problema consiste em um estudo que apresenta uma visão geral da dicotomia sobre a complexidade da minimização e da maximização do exato problema aqui estudado, demonstrando que ambos os problemas são NP-difícil para DAGs, grafos de cactos e grafos de intervalo, também demonstrou que o problema de maximização pode ser resolvido em tempo polinomial em várias classes de grafos, como árvores, grafos de blocos, grafos de intervalo adequados e particularmente para grafos de cadeia bipartida e grafos de limiar [Cohen et al. 2019].

Em casos em que cada vértice tem uma cor distinta o problema se reduz ao problema de caminho mais longo que já foi amplamente estudado na literatura. Referimos o leitor interessado a referência [Uehara and Uno 2004].

Foram encontrados outros problemas tropicais em grafos de vértices coloridos como conjuntos tropicais dominantes [d'Auriac et al. 2018], subgrafos conectados tropicais, homomorfismos tropicais e combinações tropicais [Cohen et al. 2017].

\section{Problema Proposto}

Considere $G^{C}=(G, C)$ um grafo $G=(V, A)$ com uma coloração $C: V \rightarrow \mathcal{C}$, onde $V$ é o conjunto de vértices do grafo, $A$ é o conjunto de arestas do grafo e $\mathcal{C}$ o conjunto de cores $\left\{c_{1}, \ldots, c_{k}\right\}$ utilizadas pelo grafo, ou seja, $k$ é um inteiro positivo que representa o número de cores deste conjunto. Considere também $G_{w}=(G, C, w)$, um grafo $G^{C}$ colorido nos vértices e uma função peso $w: A \rightarrow \mathbb{R}$, em que cara aresta de $A$ está associada a um número real $w(e)$ que refere-se ao peso de $e$. Neste artigo, consideramos apenas caminhos simples, ou seja, nenhum vértice é visitado mais de uma vez.

Neste problema temos duas versões, uma de minimização que é o problema de caminho tropical mínimo (STPP) e uma de maximização que é o problema de caminho tropical máximo (MTPP). Formalmente descritos:

Problema do caminho tropical mínimo (STPP). Dado um grafo ponderado colorido nos vértices $G_{w}=(G, C, w)$ e dois vértices $s$ e $t$, o problema consiste em encontrar um caminho $P$ de $s$ a $t$, que contenha todas as cores de $\mathcal{C}$ e a soma dos pesos das arestas de $P$ seja o menor peso possível. Nesta versão é permitido a repetição de cores e todas as cores de $\mathcal{C}$ ocorrem em $P$.

Problema do caminho tropical máximo (MTPP). Dado um grafo colorido nos vértices $G^{C}=(G, C)$, o problema consiste em encontrar um caminho $P$ com o maior número de cores de $\mathcal{C}$. Nesta versão não é permitido a repetição de cores no caminho, logo é possível que, em uma solução ótima, nem todas cores de $\mathcal{C}$ apareçam na solução.

A versão de decisão do TPP é NP-difícil, ou seja, não é conhecido um algoritmo de tempo polinomial para solução do problema. O plano se inicia com a modelagem do problema como um problema de programação linear inteira. Uma linguagem de programação deverá ser adotada juntamente com um pacote de otimização para implementação do modelo. No nosso trabalho o pacote adotado será o Gurobi Optimizer.

É sabido que obter solução 0/1 (binária) para problemas NP-difícil é extremamente custoso. Uma técnica da combinatória poliédrica consiste em relaxar o modelo, retirando a condição de integralidade e associando algoritmos de separação e heurísticas. Em se tratando de algoritmos de separação, quando encontrados são bem promissores na 
convergência do modelo pois eliminam soluções fracionárias em tempo polinomial. Comumente, em otimização combinatória, tais algoritmos de separação são incorporados às técnicas de branch-and-bound, branch-and-cut e relaxação lagrangiana que representam boas estratégias para obter solução ótima para para instâncias de grande porte.

Os experimentos computacionais serão realizados em instâncias reais, que representam por exemplo, interação entre proteínas, mapeamento de doenças, escalonamento de tarefas. Tal procedimento é extremamente vantajoso na análise do comportamento do resultados. Instâncias de grande porte, neste contexto, são instâncias não solucionadas diretamente pelo pacote de otimização em um limite de tempo e que dificilmente seria resolvida por qualquer outro pacote devido ao número exponencial de restrições criadas pelo modelo.

\section{Resultados Esperados}

Com uma boa formulação para o problema proposto e com algumas técnicas comumente usadas para tratar problemas computacionalmente difíceis, acreditamos que a associação com os algoritmos citados, comprovados na literatura apresentar alto desempenho, serão cruciais para obtenção de solução ótima para instâncias reais e de grande porte. A ideia é também poder analisar o comportamento do problema segundo algumas variações de classes de grafos, tais como, quantidade de cores na coloração, grau máximo de um vértice qualquer no grafo de entrada entre outros.

\section{Referências}

Bondy, J. A., Murty, U. S. R., et al. (1976). Graph theory with applications, volume 290. Macmillan London.

Cohen, J., Italiano, G. F., Manoussakis, Y., Thang, N. K., and Pham, H. P. (2019). Tropical paths in vertex-colored graphs. Journal of Combinatorial Optimization, pages 1-23.

Cohen, J., Manoussakis, Y., Phong, H., and Tuza, Z. (2017). Tropical matchings in vertexcolored graphs. Electronic Notes in Discrete Mathematics, 62:219-224.

Corel, E., Pitschi, F., and Morgenstern, B. (2010). A min-cut algorithm for the consistency problem in multiple sequence alignment. Bioinformatics, 26(8):1015-1021.

d'Auriac, J.-A. A., Bujtás, C., El Maftouhi, A., Karpinski, M., Manoussakis, Y., Montero, L., Narayanan, N., Rosaz, L., Thapper, J., and Tuza, Z. (2018). Tropical dominating sets in vertex-coloured graphs. Journal of Discrete Algorithms, 48:27-41.

Marx, D. (2004). Graph colouring problems and their applications in scheduling. Periodica Polytechnica Electrical Engineering, 48(1-2):11-16.

Sikora, F. (2012). An (almost complete) state of the art around the graph motif problem. Université Paris-Est Technical reports.

Uehara, R. and Uno, Y. (2004). Efficient algorithms for the longest path problem. In International symposium on algorithms and computation, pages 871-883. Springer.

West, D. B. et al. (2001). Introduction to Graph Theory, volume 2. Prentice hall Upper Saddle River. 